\title{
Estrategias inclusivas: atención al estudiantado con diversidad en educación básica, experiencia del profesorado hondureño
}

\author{
Inclusive strategies: attention to diverse students in primary education, the \\ experience of Honduran teachers
}

\section{Estratégias inclusivas: atenção a alunos diversos na educação básica, a experiência dos professores hondurenhos}

\author{
Percy Mejia-Elvir \\ Universidad Nacional Autónoma de Honduras \\ Tegucigalpa, Honduras \\ perzy100@gmail.com \\ (D) ORCID: http://orcid.org/0000-0003-3681-3030
}

Recibido - Received - Recebido: 15 / 09 / 2021 Corregido - Revised - Revisado: 12 / 11 / 2021 Aceptado - Accepted - Aprovado: 25 / 11 / 2021

DOI: https://doi.org/10.22458/ie.v24i36.3913

URL: https://revistas.uned.ac.cr/index.php/innovaciones/article/view/3913

\begin{abstract}
Resumen: El manuscrito presenta experiencias en estrategias y metodologías de enseñanza-aprendizaje del personal académico docente hondureño en la inclusión educativa de la niñez con necesidades educativas especiales. La unidad de análisis estuvo constituida por diez personas que tuvieran experiencia en la inclusión en nivel primario y básico educativo. El enfoque utilizado fue cualitativo, mediante un diseño de tipo fenomenológico; los instrumentos utilizados para la obtención de la información fueron dos: el primero, la entrevista narrativa, y el segundo, el grupo focal, administrándose un guion de once preguntas en la entrevista y diez en el grupo focal. Los resultados permitieron entender que sobresalen las estrategias cooperativas y participativas, a fin de armonizar sus habilidades y las relaciones sociales en el aula. Aunque no existe una formación sólida en este ámbito, se recurrió a la capacidad creativa e inventiva para configurar ambientes inclusivos. En conclusión, los apoyos para la atención a la diversidad son minúsculos, se sugiere realizar otros estudios relacionados con las ayudas que se otorgan en los planteles educativos para perfeccionar las habilidades en la atención a la diversidad.
\end{abstract}

Palabras claves: Educación básica, educación inclusiva, estrategias educativas, necesidades educativas especiales, personal académico docente, educación.

Abstract: The manuscript presents experiences in teaching-learning strategies and methodologies of Honduran academic teaching staff in the educational inclusion of children with special educational needs. The analysis unit consisted of ten people who had experience in inclusion at the primary and basic educational levels. The approach was qualitative, through a phenomenological type design; the instruments used to obtain the information were two: first, the narrative interview, and second, a focus group. We applied a script of eleven questions in the interview and ten in the focus group. The results allowed us to understand that cooperative and participatory strategies stand out to harmonize their skills and social relationships in the classroom. Albeit there is no solid training in this area, creative and inventive skills were used to configure inclusive environments. In conclusion, the supports for the attention to diversity are minuscule. It is suggested to carry out other studies related to the aids granted in the educational establishments to improve skills regarding attention to diversity.

Keywords: Primary education, inclusive education, educational strategies, special educational needs, academic teaching staff, education.

Resumo: Este manuscrito apresenta experiências de estratégias e metodologias de ensino-aprendizagem de docentes acadêmicos hondurenhos na inclusão educacional de crianças com necessidades educacionais especiais. A unidade de análise foi composta por dez pessoas com experiência em inclusão no ensino fundamental e médio. Foi empregada uma abordagem qualitativa, através de um desenho de tipo fenomenológico. Os instrumentos utilizados para a obtenção das informações foram dois: o primeiro, a entrevista narrativa, e o segundo, o grupo focal, aplicando um roteiro de onze perguntas na entrevista e dez no grupo focal. Os resultados permitiram compreender que as estratégias cooperativas e participativas destacam, de modo a harmonizar suas competências e relações sociais nasala de aula. Embora não exista uma formação sólida nesta área, foram utilizadas competências criativas e inventivas para configurar ambientes inclusivos. Em conclusão, os suportes 
para a atenção à diversidade são minúsculos, sugere-se a realização de outros estudos relacionados com os apoios concedidos nos estabelecimentos de ensino para melhorar as competências na atenção à diversidade.

Palavras-chave: Educação básica, educação inclusiva, estratégias educacionais, necessidades educacionais especiais, corpo docente acadêmico, educação.

\section{INTRODUCCIÓN}

De acuerdo con el Análisis Diagnóstico del Sistema Nacional de Educación de Honduras (2017) se enfrenta un profundo desafío, pues del anterior estudio se obtiene que, en el país, para el año 2015, había un total de 63148 menores en situación escolarizada, de los cuales un 4\% a nivel nacional de quienes reflejaban problemas de aprendizaje requerían atención especial, así como docentes debidamente calificados para asegurar que esos problemas no se convirtieran en restricción permanente que terminase expulsándoles y profundizando las tasas de exclusión y repitencia.

Otro escenario complejo que se pone de manifiesto es una insuficiente formación docente en las habilidades sobre la atención a la diversidad y la manera de intervenir ante los problemas de aprendizaje que permita optimizar el trabajo y lograr el éxito del estudiantado. Según Fermin (2014), el personal académico docente perfila carencias en las competencias de mediación del proceso de enseñanza-aprendizaje, no son adquiridas durante su formación universitaria, especialmente en la atención de la niñez escolarizada con diversidad. En consecuencia, se requiere mayor formación para el acompañamiento, orientación y mediación pedagógica a partir de estrategias y metodologías de inclusión educativa.

Sin embargo, después de la aprobación de la Ley Fundamental de Educación (2012) en Honduras, se ha dado un reconocimiento significativo en proveer espacios más inclusivos y equitativos, que tratan de garantizar un mayor acceso de justicia social a toda la niñez escolarizada. Además, destaca que las personas en situación de vulnerabilidad (personas con discapacidad, necesidades educativas especiales y talentos excepcionales), todo proceso educativo debe fundarse en principios de equidad e inclusión desde un enfoque de derechos humanos (Acuerdo, 1365-SE-2014).

En efecto, a pesar de impulsar el incremento de los derechos educativos, en el caso de la escuela pública el modelo pedagógico predominante continúa bajo un conjunto de prácticas que replican lo uniforme, desvinculándose de la diversidad de los contextos sociales, culturares y las particularidades humanas, desarrollando un proceso de exclusión educativa.

Quienes han investigado esta temática con motivo de entender y mejorar los planteles educativos, en varios trabajos han encontrado la presencia y ausencia de metodologías y estrategias para la atención a la diversidad. Atendiendo a este aspecto, Martínez (2011) llevó a la práctica un estudio cualitativo sobre la aplicación de estrategias, que promueven el desarrollo del proceso de inclusión educativa de primer y segundo ciclo de nivel básico; identificó que el planeamiento de la clase no toma en cuenta las necesidades educativas especiales del estudiantado y sus características demandan formas dinámicas y participativas.

Por su parte, Rodríguez (2013) realizó un estudio cuya finalidad se orientó en conocer cómo se utilizan los recursos humanos y materiales en la práctica inclusiva. Uno de los hallazgos suministrados, resalta la importancia entre la colaboración e inclusión, "se pudo observar como el docente de primaria tiene una visión abierta hacia la inclusión y educación especial, colabora y realiza las actividades pertinentes que permiten que la inclusión se dé" (p.92). Por el contrario, existen estudios como el de Abascal (2017), donde se sostiene que el alto ausentismo de la niñez en situación escolarizada está relacionado con las estrategias que implementa el personal académico docente en la enseñanza diversificada que constituye un problema e invita a la búsqueda de soluciones. 
Carbajal (2017) llevó a cabo un estudio concerniente a estrategias metodológicas para el cambio de actitudes docentes hacia la inclusión de estudiantes con habilidades diferentes en nivel primario del distrito de Lambayeque, Colombia. Se detectó que en las dimensiones conocimiento afectivo y conductual el personal académico docente no tiene conocimiento de inclusión educativa y de las características de los estudiantes con habilidades diferentes, tampoco desarrollan estrategias inclusivas para el cambio de estas actitudes. Por otra parte, Haspiel (2017) mencionó que la forma de concretar la práctica de la inclusión debería de reafirmar los incluyentes desde la misma sociedad, puesto que, es incluyente y es pertinente, el hecho de considerar estrategias que estimulen la sensibilización de todos los actores vinculados con la institución educativa.

En cuanto a la práctica docente, Paz (2019) indagó el tipo de competencias docentes que se podría desarrollar para atender a la diversidad en la Educación Superior Pública de Honduras. Concluye que se está en la obligación de transformar la práctica educativa, pensarla y repensarla y propone una serie de habilidades que se deberían atesorar para la atención a la diversidad, conforme a una planificación centrada en el estudiantado, ser comunicativo, implementar metodologías que promuevan el respeto y cooperativismo, pensamiento reflexivo, etcétera.

Ahora bien, Córdoba, Lara y García (2017) destacan que hay efectos positivos sobre la labor del personal académico docente, en preparar y organizar a priori metodologías lúdicas que desarrollen ambientes estimulantes, significativos hacia el progreso académico, cultura y espiritual del estudiantado. Estas se deben ajustar a sus necesidades y ser apropiadas para la expresión del buen vivir atendiendo la participación de diversos actores.

Por otra parte, Guerrero (2012) plantea que, desde la atención educativa para la inclusión, se valoran los procesos y estrategias metodológicas activas, constructivas y participativas que movilicen la capacidad mediadora de las personas, y aprovechen el valor añadido de las diferencias que amplíen el potencial de conocimiento y aprendizaje. Algunas citadas para un contexto inclusivo son las estrategias de resolución interactiva de problemas, las tutorías entre pares, los centros de interés, el estudio de caso, las tertulias dialógicas, el aprendizaje por descubrimiento, entre otras.

En concreto, se busca la manera de alcanzar un adecuado clima escolar a través de experiencias de éxito que propicien mayores expectativas de progreso, garantizar la igualdad y eliminar las barreras de diferente índole en el estudiantado con necesidades educativas especiales, y sin estas. En ese tenor, la inclusión contiene atributos que emergen como herramientas para ser más responsable ante la diversidad del estudiantado, al margen de sus origen, interés, experiencia, conocimiento y capacidad (Booth y Ainscow, 2015).

Es indiscutible que este paradigma ha impactado en la educación, experimentando un cambio significativo en el proceso de mediación del conocimiento y habilidades, lo que ha replanteado el sentido y naturaleza de los centros educativos como institución social y de formación. Análogamente, en adquirir una nueva visión pedagógica, para hacerle frente a este reto de necesidades educativas en la llamada nueva sociedad. Bajo esta óptica, la educación inclusiva como horizonte, se transforma en respuesta de la atención a la diversidad y está cada vez más ganando terreno como elemento desafiante que rechaza todo aquello que promueva cualquier tipo de exclusión en la educación (González, 2008).

Igualmente, según Gonzáles, Martin, Flores, Jenario Castro y Gómez (2013) la diversidad representa un proceso enriquecedor como potencial para fortalecer la práctica docente, por lo cual el rol de las escuelas es ofrecer herramientas acordes a sus características particulares, de manera que el estudiantado se sienta acogido, seguro y convencido que sus metas serán cumplidas. Se estima que el personal académico docente con experiencia en atención a la diversidad es poseedor de un caudal de conocimientos sobre prácticas que han sido efectivas, y que, al compartir sus propias vivencias, pueden conciliar las tenciones entre la teoría y la práctica para construir verdaderos programas educativos (Howe \& Arimoto, 2014). 
Una práctica inclusiva está orientada a atender las necesidades educativas para las personas con barreras en el aprendizaje, permitiendo su participación en distintos escenarios donde se valoren sus capacidades, con el afán de construir una comunidad solidaria y de pertenencia (Moliner, Sanahuja y Benet, 2017).

Entre tanto, Muntaner (2014) delimita una serie de acciones, orientadas a la actuación docente en la educación inclusiva acerca de aspectos metodológicos que corresponden a la organización del aula, el agrupamiento del estudiantado en gestionar la participación y el aprendizaje sin discriminación ni exclusiones; un currículo inclusivo abierto, amplio, diferenciado y riguroso con base a experiencias y actividades que se adapten a cada estudiante; una planificación para lo heterogéneo.

Al respeto, el personal académico docente tiene la obligación de reflexionar sobre sus prácticas y metodologías, a fin de verificar si se está promoviendo una enseñanza de calidad que alcance a todo el estudiantado, siendo necesario realizar cambios significativos para lograr la inclusión. Por su parte, el Diseño Universal de Aprendizaje (DUA) es otro insumo innovador que combina un enfoque inclusivo, mediante las teorías del aprendizaje, las tecnologías y los avances en neurociencia (Pastor, 2018). Para su implementación se deben proporcionar múltiples formas de implicación, es decir, se debe despertar la motivación, pero tomando en cuenta el tipo de tarea en el contexto que se ejecuta. También se deben utilizar múltiples formas de presentación de la información, en especial, proveer diferentes modos de acción y expresión, teniendo en cuenta las modalidades de aprendizaje del estudiantado e interacción con la información.

A partir de la revisión de la literatura se puede observar que existen vacíos de conocimiento sobre metodologías y estrategias que acompañen el trabajo docente, que deriven desde las mismas vivencias de profesionales educativos con experiencia en la atención a la diversidad. Además, se pone en evidencia que el personal académico docente posee escasa información en temas relacionados con la inclusión educativa, lo que genera el empleo de inadecuadas estrategias para garantizar el derecho escolar sin distinción.

El presente estudio se plantea como objetivo analizar las experiencias en estrategias y metodologías de enseñanza-aprendizaje del personal académico docente hondureño en la atención educativa, para favorecer la inclusión de la niñez con necesidades educativas especiales. Para ello se siguen los presentes objetivos específicos: a) valorar las experiencias en atención educativa que han sido exitosas en la atención de la niñez con necesidades educativas especiales por parte del personal académico docente; b) identificar estrategias empleadas por el personal académico docente, en atención educativa que faciliten las prácticas pedagógicas; c) determinar metodologías de mediación pedagógica utilizadas por el personal académico docente en atención educativa para la niñez con necesidades educativas especiales.

\section{MATERIALES Y MÉTODOS}

Se optó por un enfoque de investigación de corte cualitativo con perspectiva hermenéutica. Este trata de acercarse al mundo de allí afuera, analizando la experiencia de las personas a partir de sus historias de vida o prácticas (cotidianas o profesionales), conforme a interacciones o comunicaciones, mientras se producen (Flick, 2015). Era necesario obtener una imagen de las prácticas educativas a lo largo del tiempo (biografías) y los efectos que tuvieron.

En congruencia, se utilizó un diseño de tipo fenomenológico, lo que resulta esencial, dado que se tiene como propósito indagar en las prácticas, vivencias y situaciones para la atención educativa, orientado a poder obtener la significatividad de sus experiencias y determinar las estrategias pedagógicas inclusivas.

Diez personas participaron en el estudio, que se dividieron en dos grupos acordes a los instrumentos utilizados, seis para las entrevistas y cuatro en el grupo focal. Se seleccionaron mediante la concepción 
de informantes claves, a partir de sus vivencias y capacidad de empatizar convirtiéndose en una fuente importante de información (Robledo, 2009). La tabla 1 se describe algunos de sus datos.

TABLA 1

Datos de participantes del estudio en las entrevistas y grupo focal

\begin{tabular}{cccc} 
Códigos entrevistas & Sexo & Experiencia & Grado académico \\
P.E. 1 & F & 18 años & Licenciatura \\
P.E. 2 & F & 45 años & Licenciatura \\
P.E. 3 & F & 15 años & Maestría \\
P.E. 4 & F & 36 años & Licenciatura \\
P.E. 5 & F & 7 años & Licenciatura \\
P.E. 6 & F & 23 años & Licenciatura \\
Códigos grupo focal & Sexo & Experiencia & Grado académico \\
P.E. 1 & F & 27 años & Licenciatura \\
P.E. 2 & F & 32 años & Licenciatura \\
P.E. 3 & F & 30 años & Licenciatura \\
P.E. 4 & F & 19 años & Licenciatura \\
\hline
\end{tabular}

Los criterios para la selección fueron los siguientes: a) personal académico docente en los niveles primario o básico, b) que tuviesen más de cinco años de experiencia en el abordaje de la niñez con necesidades educativas especiales, y c) que tuvieran anuencia plena para participar en el estudio.

Por lo que se refiere a las entrevistas, se desarrolló un guion de once preguntas narrativas, Alheit (2013) manifiesta que se basa en una teoría concreta sobre la narración de historias abiertas. El proceso se inició contactándoles por medio de llamadas telefónicas y solicitando su participación en el estudio. Posteriormente, se ejecutaron haciendo uso de la aplicación Zoom, bajo un ambiente de confianza, indagando en su formación, trayectoria y vivencia de la temática. Se registraron en video con el consentimiento informado de cada persona entrevistada, organizadas y tuteladas por el investigador con una duración de 60 minutos.

Para el grupo focal, el proceso se realizó con la misma aplicación, conforme a un guion de nueve preguntas. Las entrevistas fueron almacenadas en video con el consentimiento de cada persona. Este procedimiento permitió profundizar en sus experiencias e identificar las apreciaciones y situaciones sobre la implementación de estrategias, en torno a la diversidad del aula y las barreras del aprendizaje. El investigador se ocupó del procedimiento, efectuándose dos sesiones con un plazo de 55 minutos cada una.

Para garantizar la confiabilidad y validez de los instrumentos, se aplicaron la prueba piloto y el criterio de validez de contenido. Tras la ejecución de los procesos, se transcribieron los videos y se desempeñó la elaboración de un informe individual por cada persona entrevistada y las dos reuniones del grupo focal, teniendo en cuenta las categorías derivadas de los objetivos del estudio.

Una vez realizada la fase de transcripción, se analizó y revisó la información, a fin de realizar registros narrativos y después llevar a cabo, el análisis que permitiera alcanzar un sistema de asociación y correlación, entre las entrevistas y las respuestas del grupo focal. Este mecanismo fue llevado a la práctica a través de la triangulación, debido a que confiere un marco mayor de objetividad de un fenómeno social; además, es resultante de la retroalimentación de diferentes acercamientos y existen interpretaciones de los datos desde varios ángulos (Izacara, 2014). 


\section{DISCUSIÓN DE RESULTADOS}

Al analizar la experiencia en estrategias y metodologías del personal académico docente para favorecer la inclusión, el hallazgo principal permitió entender que sobresalen las actividades cooperativas y participativas, pues fueron útiles en la armonización de las habilidades y relaciones sociales en el aula. Asimismo, hay acciones que no derivaron de pautas externas de formación, tanto que se recurrió a la innovación y creatividad para la configuración de ambientes inclusivos. Uno de los componentes claves, es la estructuración de actividades que despiertan diversos canales sensoriales, facilitando una restructuración cognitiva y, a la vez, el desarrollo de otras habilidades.

En cuanto a los resultados de las entrevistas y el grupo focal, se agruparon por categorías de análisis del estudio que se generaron de los objetivos. Igualmente, se presentan en los apartados algunas tablas para una mejor comprensión.

\section{Experiencia del personal académico docente en la atención educativa}

Medular en el análisis es la referencia que permite comprender la experiencia docente en la atención a la diversidad que fue satisfactoria verbalizada en las entrevistas.

TABLA 2

Resultados de los aspectos más sobresalientes en las entrevistas relacionado con la experiencia del profesorado.

\section{Subcategoría: Trayectoria en la enseñanza inclusiva:}

- Experiencia previa en la labor áulica antes de su formación en la atención a la diversidad (P.E. 1, 2, 3, 4 y 6)

- Construcción de habilidades a partir de distintas vivencias en intervención sobre casos de niñez con NEE (P.E. 1, 2 y 5)

Subcategoría: Vivencias en la atención educativa:

- Prácticas centradas en las características del estudiantado (P.E. 1, 2, 4, 5 y 6)

- Implicación de distintos miembros (estudiantado, padres, otros profesionales) para superar barreras y dificultades en la atención educativa (P.E. 2 y 5 )

- Intervención a partir de ideas no convencionales (creatividad e innovación) (P.E. 1, 2 y 4)

Fuente: Elaboración propia.

Como se puede observar en la tabla 2, las entrevistadas, en su mayoría, revelaron que, experimentar situaciones previas antes de la formación docente ha afianzado su implicación en la atención a la diversidad. Una persona entrevistada dijo: "Hablo del nivel primario, mis tres primeros años, estuve trabajando en un proyecto en el Centro de Innovación e Investigación Pedagógica que se llama proyecto Integración" (P.E. 3).

Con el fin de consolidar el compromiso con el apoyo hacia el estudiantado, se adopta la conciencia de reformar la estructura de desigualdad que persiste en los centros educativos. No obstante, hay poca evidencia en las entrevistas de que se hayan adquirido habilidades en espacios de educación formación formal o alternativa antes de estas experiencias, lo que denota la falta de preocupación en el sistema educativo por equipar habilidades para la atención del estudiantado con habilidades diferentes. Se concuerda con Fermin (2014), ya que no se evidencian para la atención a la diversidad, desde la formación universitaria.

Se ilustra como resultados que tres de las personas entrevistadas manifestaron haber experimentado procesos de intervención educativa en distintos casos; lo que fue sustancial para la adquisición de habilidades y construcción de espacios pedagógicos inclusivos, por ejemplo: "Sí he tenido en mis aulas de todos los ángulos. En la escuela yo he tenido niños con necesidad especial principalmente con autismo o alguna discapacidad motora" (P.E. 3). Es alentador comparar lo anterior con los datos de Howe \& Arimoto 
(2014) que, a partir de haber experimentado diversas circunstancias con la atención a la diversidad, permitió adquirir una base de conocimiento, la cual colabora y dirige las acciones que sean pertinentes a cada caso, entrelazando la teoría y práctica.

Un hallazgo importante fue al que se refirieron dos de las personas entrevistadas, sobre el compromiso de distintos actores para desarrollar acciones y lograr el éxito en la inclusión, así como el perfeccionamiento de habilidades en el estudiantado y llegar a los objetivos del plan educativo: "Buscamos una estrategia con los papás, porque aquí hay que tener no solo lo que dicen, hay que inventar cosas diferentes" (P.E 4). Este factor podría explicar la responsabilidad de diferentes miembros, sobre la dedicación en trabajar en manera conjunta como protagonistas dinámicos, activos y participativos que tratan de eliminar las barreras.

La tabla anterior proporciona datos significativos. Se mencionó que las actividades de mediación pedagógica vivenciadas, evidencian una cualidad como la creatividad e innovación para dar solución a los problemas en la intervención. Lo confirma la persona entrevistada uno: “Una simple suma que dos más dos es cuatro yo lo trabajo con palias, yo lo trabajo con los dedos y la otra opción, cuando estábamos en la clase de español le decía "vamos a enlazar con matemáticas" (P.E 1).

En función de todo lo mencionado, se infiere que estructurar diferentes rutas de acción educativa facilita solventar las situaciones. Se adecua el trabajo a partir de ideas no convencionales que son claves para encontrar formas constructivas de solución, armonizando las oportunidades de acceso de manera igualitaria a todo el estudiantado. Aquí denota la capacidad de resiliencia del personal académico docente, la atención a la diversidad contribuye de manera significativa en potenciar y nutrir el quehacer docente, puesto que hay un replanteamiento de su práctica constantemente y lleva a posibilitar insumos acordes a las particularidades del estudiantado (González et al. 2013). De manera que se siente acogido, seguro y que sus oportunidades de acceso son iguales que al resto de sus pares.

\section{Estrategias empleadas por el personal académico docente en atención educativa que facilitan las prácticas pedagógicas}

Como se muestra en la tabla 3, lo relacionado con estrategias en la enseñanza inclusiva, las actividades, desde la perspectiva docente fue excelente haber empleado la tutoría entre pares, para reducir las desigualdades del estudiantado más vulnerable: "Le digo al compañerito: 'Tú ya terminaste la tarea'; hablo con él y le digo que le enseñe" (P.E 2). En coherencia con lo anterior, existen dos estrategias notables, las adecuaciones curriculares y títeres, estas fueron mencionados por tres de las entrevistadas. Estratégicamente este hallazgo, apoya aún más la idea que la enseñanza inclusiva debe ser participativa, cooperativa y abierta, a causa de que maximiza el potencial del estudiantado, considerando sus intereses, saberes previos y capacidades. Se coincide que la inclusión es factor desafiante que rechaza la idea de la exclusión en el terreno educativo (González, 2008).

Sorprendentemente, se encontró que el agrupamiento de equipos, materiales con relieves, videos y láminas aumentadas se describieron con menor grado: “...usamos también bastantes videos, que tengan un lenguaje de señas que muestren el contenido como tal" (P.E 6). A su vez, actividades motoras se refirieron una vez. Se evidencia lo unilateral que es pensar en acciones inclusivas separadas de lo colectivo, por lo tanto, se sugiere el acompañamiento de los procesos a lo interno del aula de clases por el personal administrativo, no de forma fiscalizadora, sino para implicar procesos más integrales en estos escenarios. 
Tabla 3

Resultados más sobresalientes en las entrevistas relacionados con las estrategias empleadas para facilitar las prácticas pedagógicas.

\section{Subcategoría: Estrategias en la enseñanza inclusiva}

- Tutorías en pares (P.E. 1,2 y 3)

- Láminas con detalles resaltados (P. E. 4 y 5 )

- Videos con lenguaje de señas (P.E. 3 y 6 )

- Agrupamiento por equipos (P.E. 3)

- Actividades motoras, julajoo y encostalado (P.E. 2)

- Materiales con relieves (P.E. 5 y 6 )

- Sistemas alternativos y aumentativos de comunicación (P.E. 2, 3, 5 y 6)

- Adecuaciones curriculares (P.E. 2, 3 y 5)

- Títeres (P.E. 1, 2 y 6 )

\section{Subcategoría. Recursos y herramientas en la atención educativa:}

- Material reutilizable (cajas, papel, etcétera) (P.E. 1 y 5 )

- Tecnología braille (P.E 5 y 6 )

- Juegos por medio de las Tecnologías de la Información (aplicaciones de celulares, proyectores) ( 3 P.E. 1, 4 y 5)

- Bloques lógicos de Dienes (P.E. 6)

- Letrero de comunicación para la niñez autista (P. E. 5)

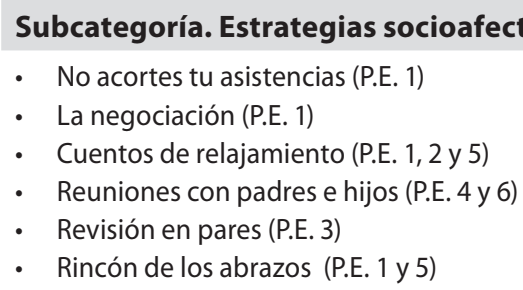

Subcategoría. Apoyos para la enseñanza inclusiva:

- Capacitación docente (P.E. 4 y 5)

- Escritura sombra (P.E. 3)

- Marcadores de diferentes colores (P.E. 4 y 6 )

Fuente: Elaboración propia.

Asimismo, se muestra en la tabla 3 que las personas entrevistadas señalaron hacer uso de distintos recursos en las acciones de inclusión. Una observación importante que se destacó fue la tecnología, según, favorece la apropiación de la información en el estudiantado: “...la tecnología nos ha venido a ayudar mucho. Ejemplo, yo he tenido en mi salón niños con autismo, [...] un recurso que me ayuda mucho es tener una computadora o tener Data Show" (P.E 5). Se acepta lo planteado por Laitón et al. (2017), de que la tecnología crea múltiples acciones convertidas en estrategias integrales, que garantizan justicia al estudiantado sin distinción. Pese a lo dicho en los resultados, es curioso darse cuenta de que el profesorado ha adoptado una actitud de renovación constante en sus competencias por sí mismos, no a partir de un programa de estímulos derivados de la Administración Macro Educativa.

Al respecto de estrategias socioafectivas, la tabla 3 muestra un hallazgo sólido, se dijo que en las aulas ha tenido un resultado importante las actividades: "...por medio de cuentos, [...] algo está pasando entonces hay que favorecer. Entonces buscamos esa historia relacionada" (P.E 5). Un segundo factor que se citó fue: “...la co-revisión, él le revisa al compañero. Entonces los niños [...] de repente me dicen yo le voy ayudar a fulano (sic)" (P.E 1).

Los dos anteriores aspectos son significativos, a la luz que las estrategias con carga afectiva permiten incrementar el nivel de asimilación de conocimiento, autoconcepto y desarrollan una actitud positiva sobre sí mismo; además, nutre valores como la empatía, justicia e igualdad, se fomenta la participación y 
adaptación social. El apropiado manejo de las emociones tiene la intención de intensificar la motivación del estudiantado, pero se debe tener mucha cautela al decidir implementar actividades si se desconoce cómo enlazar las actividades, mediante un currículo transversal.

Respecto a apoyos para la enseñanza inclusiva, la tabla muestra diversos aspectos a tomar en cuenta. Sin embargo, se mencionaron una reducida cantidad de recursos innovadores que emplea el personal académico docente en la atención a la diversidad, sin duda este tema podría ser objeto de un escrutinio riguroso de medios para el aprendizaje y enseñanza inclusiva. Es necesario seguir trabajando acerca de cuáles son las ayudas proporcionadas para la enseñanza inclusiva en los planteles educativos.

\section{Metodologías de mediación pedagógica utilizadas por el personal académico docente en atención educativa}

Cuando se les preguntó a las personas entrevistadas: ¿Qué metodologías han funcionado para atender al estudiantado con NEE?, las respuestas fueron múltiples. Con ello se establecen una serie de actividades que no responden a un único aspecto.

Tabla 4

Resultados más sobresalientes en las entrevistas relacionados con las metodologías de mediación pedagógica.

\section{Subcategoría. Procedimientos de enseñanza:}

- Atención individualizada (P.E. 1, 2 y 5)

- Aprendizaje situado/experiencial (P.E. 1)

- Estimulación sensorial, mediante diferentes canales de información (P.E. 3 y 5 )

\section{Subcategoría. Practicas inclusivas:}

- Destinar pequeñas responsabilidades al estudiantado con NEE o deficiencias/ asistente docente (P.E. 1 y 4 )

- Estudio de casos (P.E. 3 y4)

- Equipos de minutos (P.E. 3)

- Equipos de expertos (P.E. 3)

- lluvia de ideas (P.E. 3)

- Aprendizaje por resolución de problemas (P.E. 3 y 4)

- Metodología ABA (P.E. 5)

\section{Subcategoría. Metodologías de lecto-escritura y memoria:}

- Método de cuentos (P.E. 2 y 5 )

- Encadenamiento de palabras a través de tarjetas (P.E. 2, 4 y 5)

- Juegos por cantos, dinámicas, onomatopeyas (P.E. 1, 2 y 4)

- Rompecabezas, sopas de letras, legos (P.E. 6)

- Ejercicios de gimnasia cerebral (P.E. 3)

\section{Subcategoría. Metodologías lúdicas:}

- Dramatizaciones (P.E. 2)

- Competencias de deletreo (P.E. 4)

- Rincones de trabajo (P.E. 5)

- Adivinanzas (P.E. 1)

- Juegos de manipulación (bolitas de harina) (P.E. 1)

- El Gallo (P.E. 2)

- Dibujo con pintura (P.E. 2)

- Juego del elefante (P.E. 6)

- Bingo de colores (P.E. 4)

- Paseos lúdicos (P.E. 4 y 6 )

Fuente: elaboración propia. 
En la tabla 4 se exponen los procedimientos de enseñanza. Las personas entrevistadas relataron reiteradamente que: "la atención personalizada da mejores resultados, uno aprende a conocer mejor el estudiante a ver qué cosas pasan y atenderlo" (P.E 6). Una práctica individualizada proporciona respuesta de calidad al estudiantado sobre su interés, sus capacidades y necesidades asegurando su inclusión. Parte de las grandes críticas realizadas es la carencia de acciones en generar una cultura de apertura, a todos los miembros de la comunidad educativa, elemento que limita visibilizarles, es así que, se vuelve necesario reafirmar estrategias que estimulen la vinculación de todos los actores (Haspiel, 2017).

Otro aspecto que aparece varias veces aludido fue: "la estimulación, el manejo de los sentidos [...] me gusta que manipule las cosas" (P.E 2). Lo anterior concuerda con el DUA, para impulsar la inclusión, es necesario implementar distintas formas de presentación de la información, que permita despertar varios canales de asimilación del conocimiento (Pastor, 2018). Por el contrario, una clara desventaja de este enfoque ha sido visto como un producto de la discapacidad y no un método de enseñanza para el estudiantado en general.

Igualmente, se encontró que las actividades cooperativas y colaborativas permiten cultivar en el estudiantado la capacidad de desarrollo personal y contribuir a los objetivos de manera común, evitando ayudas por sus deficiencias mediante el trabajo en conjunto. La entrevistada tres dijo: “...todo con lo que tiene que ver con agrupamientos sensibles, acciones tutoriales..." (P.E 3). Se coincide que, resaltar procesos activos y participativos prevé un valor añadido, en ampliar las capacidades y conocimiento del estudiantado, incluso cultiva valores sociales (Guerrero, 2012). En el país se ha infravalorado la experiencia social de los centros, por lo tanto, una cuestión que necesita ser tratada son las interacciones en el aula, pues analizarla ayudaría a mejorar las condiciones colectivas de la escuela.

Como se lista anteriormente, las metodologías de lecto-escritura y memoria, la mayoría de entrevistadas relataron, que la metodología predominante en el desarrollo de la habilidad lectoescritora, es el encadenamiento de palabras por medio de tarjetas: "se desarrolla la memoria a través de juegos, juegos de palabras, frases repita dos palabras empezadas con dos palabras" (P.E 4). Con referencia a lo anterior, significa un proceder coherente bajo un ambiente controlado, ordenado, libre e inclusivo que eleva la retención de la información. No se concuerda con Abascal (2017), pues los datos arrojados evidencian que el personal académico docente implementa estrategias diversificadas, que dan cuenta el hecho de apostar por una educación inclusiva.

La tabla puntualiza que las narrativas dejaron entrever distintas acciones lúdicas llenas de armonía, la entrevistada uno narró: "...a mí me encanta mucho trabajar con dramatizaciones, que ellos dramaticen..." (P.E 1). Otra persona aseveró que: "Ios juegos son mágicos para integrar el grupo, integrar el niño, por ejemplo, utilizamos rincones de trabajo" (P.E 5).

Sobre la base de lo anterior, los aspectos se adhieren a lo que afirman Córdoba et al. (2017) acerca de los efectos positivos que repercuten al organizar actividades lúdicas, a razón que generan un ambiente estimulante hacia el progreso académico y espiritual del estudiantado, fomentando relaciones más profundas que evitan la discriminación. Aun así, estos resultados difieren de lo encontrado por Martínez (2011), quien afirma que el planeamiento de las clases no toma en cuenta las necesidades del estudiantado, por lo tanto, no se logra resolver la contradicción entre si se ejecutan acciones o no se toman en cuenta las necesidades.

A continuación, se presentan los resultados de las respuestas del grupo focal. Primero se despliega la categoría y luego las opiniones más representativas expresadas por el grupo de personas informantes en las diferentes sesiones. La siguiente tabla denota las respuestas más representativas.

En la Tabla 5 se encuentra el análisis de los datos más significativos obtenidos de las cuatro personas informantes, lo que acentúa los aspectos más preponderantes en las categorías. 
TABLA 5

Análisis de las respuestas de participantes en el grupo focal

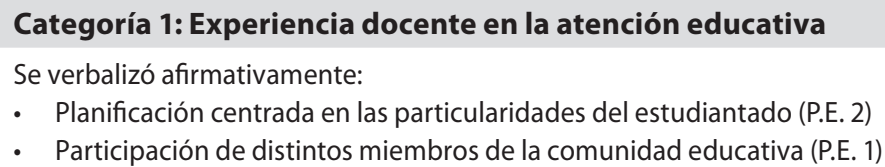

Categoría 2: Estrategias empleadas por el profesorado especialista en atención educativa que faciliten las prácticas pedagógicas

- Apoyo de autoridades educativas (P.E. 3)

- Capacitación docente (P.E. 3)

- Apoyo extraescolar fuera del centro educativo (P.E. 4)

- Interpretes (P.E. 3)

- Herramientas tecnológicas (P.E. 3 y 4 )

- Encadenamiento de palabras (P.E. 1)

- Dramatizaciones (P.E. 1 y 2 )

- Cuadros sinópticos (P.E. 4)

- Material con texturas (Profesorado E. 2)

- Marcadores de diferentes colores (P.E. 4)

- Caja calendario (P.E. 4)

- Métodos multisensoriales (P.E. 1, 2, 3 y 4)

- Trabajo en pares (P.E. 3)

Categoría 3: Metodologías de mediación pedagógica utilizadas por el profesorado especialista en atención educativa

- Actividades colaborativas y cooperativas (exposiciones) (P.E. 1)

- Cuentos (P.E. 1 y 2)

- Clave de Sil Gerald (P.E. 3)

- Refranes (P.E. 4)

- Juegos tradicionales (P.E. 3)

- Bingos (P.E. 1)

- Competencias estudiantiles (P.E. 4)

- Diseño Universal del Aprendizaje (DUA) (P.E. 4)

- Historietas con sus personajes (P.E. 2)

Fuente: elaboración propia.

\section{Experiencia del personal académico docente en la atención educativa}

1.1. Participación en primer lugar de padres de familia, participación de los mismos alumnos.

1.2. El apoyo de las autoridades, siempre uno ve la forma de cómo hacer las cosas.

1.3. Hay un aspecto importante a considerar y es hacer un trabajo en equipo [...] parte médica, parte psicológica y el abordaje de la familia.

Basándose en los datos de la tabla, se reveló una clara tendencia de experiencias hacia el fortalecimiento de las habilidades en el estudiantado, con enfoque de participación de distintos agentes educativos al proceso de atención. Los resultados son conscientes de que hay una relación intercultural y de patrones de socialización que implica el desempeño colectivo de todos los miembros, su intervención se concibe como simbólica, es pertinente que las acciones sean acompañadas con una interacción social significativa. 
Como se ha dicho, para atender a la diversidad es indispensable implementar metodologías que promuevan el cooperativismo (Paz, 2019). Mientras, el citado autor indica varias competencias sobre la labor de la atención educativa. Este estudio propone otra más, la resiliencia, atributo que resulta conveniente para superar los inconvenientes y resolver los problemas con acciones correctivas y de prevención.

\section{Estrategias empleadas por el personal académico docente en atención educativa que faciliten las prácticas pedagógicas}

2.1. Darle mucho la parte visual, por ejemplo, en las clases hay que darle muchos cuadros sinópticos...

2.2. Utilizar la tecnología, inventar el sistema con algo bonito ¡no sé! Videos.

2.3. ...cuando se les enseñe el maestro debe utilizar marcadores de diferentes colores. sobre todo, hay que trabajar mucho con material concreto, hay que implementar mucho las texturas.

La tabla resume que se observó una tendencia amplia en las respuestas de las personas informantes sobre la importancia de utilizar estrategias visuales, como actividades para mediar la enseñanza y atender al estudiantado que presenta deficiencia. Existe una influencia significativa de la introducción de elementos que ayuden al bienestar de la comunidad educativa, propiciando variadas herramientas sensoriales, con el objetivo de tener una participación más amplia, mediante el respeto a las múltiples formas que tienen las personas de lograr la comprensión.

Por otro lado, en esta explicación se debe ser muy cauteloso, debido que se tiende a pasar por alto la eficacia en producir un efecto deseado de las actividades a partir de la creatividad. Cabe resaltar que la precisión de estos elementos no debe estar ausente de una planificación sin valorar la idiosincrasia de las personas (Muntaner, 2014). Sucede que, en ocasiones se toman actividades exteriores y estas no encajan a las habilidades diferentes del estudiantado, en consecuencia, no responden a la mejora de sus capacidades. Por ende, la generación de ideas no ordinarias es imprescindible.

Asimismo, en la tabla, otro de los factores en que hubo convergencia de opinión por parte de las personas participantes como estrategia fue la tecnología.

2.6. Material tecnológico; aprovechar todos los recursos disponibles para poder presentar las lecciones.

2.7. La tecnología es muy buena en este momento.

Con base en las respuestas destacadas en la tabla, las participantes destacaron que la tecnología es un recurso de provecho para la atención educativa. Se determina que su ejecución en la mediación pedagógica favorece la apropiación del aprendizaje del estudiantado. Estudios previos (Booth y Ainscow, 2015; González, 2008) explican que la educación inclusiva pone entre dicho lo homogéneo; de ahí que el utilizar distintas estrategias de enseñanza en los planteles educativos, supera el pensamiento de lo único y allana el camino hacia una comunidad educativa inclusiva.

\section{Metodologías de mediación pedagógica utilizadas por el personal académico docente en atención educativa}

3.1. La metodología de cuentos [...] que es utilizada la fonética y la gramática.

3.2. Si hay que probarlo, lo que decía el método natural o de cuentos también. 
La tabla proporciona una visión general sobre este asunto, las personas participantes dejaron de manifiesto las bondades que ofrece el cuento como metodología de enseñanza. Por medio de esta acción, se promueven diversas capacidades en el estudiantado: surge la imaginación y la manipulación del lenguaje, en virtud se crean escenarios sociales que facilitan la capacidad de concebir ideas asociada a la construcción de textos. Lo precedente corresponde con el criterio de Terrades (2012), que plantea que, a partir del cuento, el estudiando dispone de varias capacidades relacionadas con la creatividad, la comprensión y entendimiento de los escenarios sociales que les rodea.

Por otra parte, una comprensión más amplia estaría enfocada, no exclusivamente en el desarrollo sensorial, sino que acentúa su valor en lo cognitivo por la asimilación de la información que se produce; comunicación, en el intercambio consciente y participante de diálogo; y lo social, pues se cambia la cultura de los centros educativos.

Además, la tabla sintetiza otro aspecto que emergió como estrategia de las personas participantes.

\subsection{La colaboración se cumple [... y y la recompensa es muy importante.}

\subsection{El trabajo en equipo o las actividades grupales, las competencias y las recompensas.}

Igualmente, las respuestas señalaron, que las experiencias educativas por metodologías colaborativas y cooperativas fortalecen el acompañamiento y la construcción del conocimiento entre todos los miembros, a lo interno de las aulas de clases, porque se equiparan las oportunidades educativas y la atención a la diversidad. En relación con lo mencionado, la actividad grupal fomenta el sentido social y se trabaja respetando las características del estudiantado al margen de sus dificultades. Esto implica la práctica de habilidades comunicativas y positivas, por lo que propicia el acceso a una educación de mayor calidad y sentido de igualdad (Moliner et al, 2017).

\section{SÍNTESIS Y REFLEXIONES FINALES}

Al analizar la experiencia en estrategias y metodologías del personal académico docente para favorecer la inclusión, el hallazgo principal permitió entender que sobresalen las actividades cooperativas y participativas, y que estas fueron útiles para la atención y armonización de habilidades y relaciones sociales en el aula de clases. Asimismo, hay acciones que no derivaron de pautas externas de formación, tanto que se recurrió a la innovación y creatividad para la configuración de ambientes inclusivos. Uno de los componentes claves, es la estructuración de actividades que despiertan diversos canales sensoriales, facilitando una restructuración cognitiva, a la vez el desarrollo de otras habilidades en el estudiantado.

En cuanto a la experiencia del personal académico docente puede plantearse que esta fue exitosa. Se concluyó de las entrevistas y del grupo focal que su trabajo estuvo influenciado por gozar de diversa experiencia previa en la atención, pero que las distintas situaciones y casos han contribuido a crear nuevas soluciones para responder a la diversidad. Se recomienda estructurar capacitaciones periódicas al personal académico docente, además la conformación de grupos interdisciplinarios que orienten el quehacer docente en los centros escolares. Conviene subrayar que mostraron resiliencia y, por ello, una actitud positiva empleando diferentes medios. Esto facilita la capacidad creativa e innovadora, implicando una adaptación ante las circunstancias y la solución de problemas.

Con respecto, a las estrategias empleadas para facilitar la práctica pedagógica en la atención educativa central en estas conclusiones es indicar que aquellas que mejor resultado ofrecen a la inclusión, son las participativas y cooperativas. Las primeras consisten en realzar el involucramiento de todo el estudiantado, garantizan la igualdad y están dedicadas a la comprensión de su idiosincrasia. En las segundas, su núcleo se focaliza en la interacción que cultiva valores como aceptación, empatía, justicia e igualdad 
que nutren de actitudes orientadas a derrumbar las barreras. En balance, las tutorías entre pares, dramatizaciones, equipos de minutos, equipos de expertos, alimentan actitudes positivas hacia las demás personas.

En referencia a los apoyos educativos para garantizar habilidades del estudiantado, se evidenció que el trabajo engloba diversos medios que amplían lo sensorial y esto ayuda la atención a la diversidad. Por el contrario, los hallazgos en este aspecto fueron minúsculos, a su vez, se sugiere proseguir estudios posteriores relacionados con ayudas ofrecidas a los planteles educativos, desde la administración educativa a nivel macro, acerca de la atención a la diversidad o medios utilizados por el personal académico docente en la mediación pedagógica.

En lo que concierne a las metodologías, este estudio permite determinar que se hace énfasis en aspectos como el cuento, tarjetas de palabras, el encadenamiento de palabras y otras más. Aparentemente, generan una restructuración cognitiva y, a la misma vez, incrementan habilidades comunicativas en el estudiantado que presenta habilidades diferentes. También, la evidencia apoyó la idea que las metodologías lúdicas en especial cultivan la estima, el gozo y la motivación dentro del paradigma de la educación inclusiva, lo que permite orientar al trabajo didáctico a raíz del carácter transversal en los contenidos.

\section{REFERENCIAS}

ABAsCAL, M. (2017). Las Prácticas Inclusivas en las Escuelas Primarias de Córdoba: las condiciones pedagógicas y el acompañamiento a las trayectorias escolares reales de alumnos en situación de vulnerabilidad social (tesis de maestría). Universidad Católica de Córdova, Córdova, Argentina. Recuperado de: http://pa.bibdigital.uccor.edu.ar/1418/1/TM_Abascal.pdf

Alheit, P. (2013). La entrevista narrativa. Plumilla Educativa, 10(2), 11-18. https://doi.org/10.30554/ plumillaedu.10.84.2012

Bоотн, T. y Ainscow, M. (2015). Guía para la Educación Inclusiva. Desarrollando el aprendizaje y la participación en los centros escolares. Madrid, España: Editorial Fuhem.

Carbajal, M. (2017). Programa de Estrategias Metodologías para el Cambio de Actitudes Docentes Hacia la Inclusión de Estudiantes con Habilidades Diferentes en las Instituciones Educativas del Nivel Primario del Distrito Lambayeque (tesis de doctorado). Universidad Cesar Vallejo, Lima, Perú. Recuperado de: https://repositorio.ucv.edu.pe/handle/20.500.12692/2503

Congreso de la Republica. (2012). Ley Fundamental de Educación. Diario Oficial No. 32,754. Recuperado de: https://www.se.gob.hn/media/files/leyes/LE_5.pdf

Córdoba, E., Lara, F. y García, A. (2017). El juego como estrategia lúdica para la educación inclusiva del buen vivir. Ensayos, Revista de la Facultad de Educación de Albacete, 32(1), 81-92. DOI:10.18239/ ensayos.v32i1.1346

Fermín, M. (2014). Por una educación inicial y para la diversidad: ¿conocen y atienden los maestros este reto educativo? Diversidad e Identidad en la Educación, 1(2), 13-25.

FLIck, U. (2015). El diseño de la Investigación Cualitativa. Madrid, España: Ediciones Morata.

Gonzalez. F., Martin, E., Flores, N., Jenario Rio, C., Poy Castro, P. y Gomez, M. (2013). Inclusion y convivencia Escolar analisis de la formación del profesorado. European Journal of Investigation in Health, Psychology and Education, 3(2), 125-135.

GonzALEZ, M. (2008). Diversidad e Inclusión Educativa: algunas reflexiones sobre el liderazgo en el centro escolar. Revista Electrónica Iberoamericana sobre Calidad Eficacia y Cambio en Educación, 6(2), 89-99. Recuperado de: https://revistas.uam.es/reice/article/view/5443 
GuerReRo, C, (2012). Hacia la construcción de procesos y prácticas "exclusivas": Metodologías para la intervención. Revista semestral para animadoras y educadoras sociales, (16), 1-10.

Hakspiel, C. (2017). Sistematización de la Experiencia de la Inclusión Educativa Colegio Nuevo Gimnasio (tesis de pregrado). Pontificia Universidad Javeriana, Bogotá, Colombia. Recuperado de: https://repository.javeriana.edu.co/handle/10554/39261

Howe, E. \& MAsAHIRO, A. (2014). Narrative Teacher Education Pedagogies From Across the Pacific. Emerald Insight, (22), 213-222. DOI: https://doi.org/10.1108/S1479-368720140000022014

Izacara, P. (2014). Manual de Investigación Cualitativa (1. a edición). Coyoacán, México: Ediciones Fontamara.

Laitón, E.V., Gómez, E.E., Sarmiento, R.E, y Mejía, C. (2017). Competencia de prácticas inclusivas: las TIC y la educación inclusiva en el desarrollo profesional docente. Sophia, 13(2), 82-95. DOI: https://doi.org/10.18634/sophiaj.13v.2i.502

MARTínez, N. (2011). Aplicación de estrategias de enseñanza que promueven el desarrollo de procesos de integración e inclusión educativa en estudiantes de l y ll ciclo de la Educación General Básica. Estudio de caso Centro Educativo San Juan (tesis de maestría). Instituto Tecnológico de Monterrey, Monterrey, México. Recuperado de: https://repositorio.tec.mx/handle/11285/571017

Moliner, O., Sanahuja, A. y Benet, A. (2017). Practicas Inclusivas en el Aula desde la Investigación- Acción. Valencia, España: Publicacions de la Universitat Jaume I. Recuperado de: http://repositori.uji.es/ xmlui/bitstream/handle/10234/168902/s127.pdf?sequence=1\&isAllowed=y\%C3\%A7

MUNTANER, J., (2014). Practicas inclusivas en el aula ordinaria. Revista nacional e internacional de educación inclusiva, 7(1), 63-79.

Ñaupas, H., MejíA, E., I. y Villagómez, A. (2014). Metodología de la investigación Cuantitativa - Cualitativa y Redacción de la Tesis (4. a edición). Bogotá, Colombia: Ediciones de la U.

PASTOR, C. A. (2018). Diseño Universal para el Aprendizaje: un modelo teórico para una educación inclusiva de calidad. Recuperado de: https://www.educacionyfp.gob.es/dam/jcr:c8e7d35c-c3aa-483dba2e-68c22fad7e42/pe-n9-art04-carmen-alba.pdf

PAZ, E. (2019). Una indagación narrativa autobiográfica sobre las competencias docentes del profesorado universitario para la atención a la diversidad en una universidad pública de Honduras (tesis de maestría). Universidad Católica del Maule, Talca, Chile.

Secretaria de Educación de Honduras. (2017). Informe Sistema Educativo Hondureños en Cifras Periodo Académico 2014-2016. Recuperado de: https://www.se.gob.hn/media/files/articles/201711_ usinieh_informe_estadistico_2014_2016_hbha1lq.pdf

TeRRADES, L. (2012). Aprender a leer y escribir contando cuentos en Educación Infantil (tesis de pregrado). UniversidadInternacionaldelaRioja,Logroño,España.Recuperadode:https://reunir.unir.net/bitstream/ handle/123456789/841/2012_09_17_TFG_ESTUDIO_DEL_TRABAJO.pdf?sequence=1\&isAllowed=y

RoBLEDo, J. (2009). Observación participante: informantes claves y rol del investigador. Nure investigación, (42), 1-4. Recuperado de: https://www.nureinvestigacion.es/OJS/index.php/nure/article/ view/461

RodRIGUEZ, B. (2013). La inclusión educativa como práctica cotidiana a través de la colaboración entre los actores de una escuela primaria (Tesis de maestría). Instituto Tecnológico de Monterrey, Monterrey, México. Recuperado de https://repositorio.tec.mx/handle/11285/626493 